\section{Evaluation of the Clinical Practice of Shoulder Examination among Ten Experienced Shoulder Surgeons}

\section{Ann Ganestam ${ }^{1 *}$, Mikkel L Attrup ${ }^{2}$, Per Hølmich ${ }^{2}$ and Kristoffer W Barfod ${ }^{2}$}

${ }^{1}$ Department of Orthopaedic Surgery, Copenhagen University Hospital, Hvidovre, Denmark

${ }^{2}$ Department of Arthroscopic Surgery, Copenhagen University Hospital, Amager, Denmark

\begin{abstract}
Context: Shoulder problems constitute a major socioeconomic problem with lifetime prevalence up to $66.7 \%$. To aid the diagnosis more than 184 tests have been described. Although a standardized guideline for a complete shoulder examination is not available, we hypothesize that experienced shoulder surgeons have clear preferences among Shoulder Physical Examination (SPE) tests.

Aim: The aim of this study was to identify the most used SPE tests for 12 selected shoulder pathologies and evaluate the usefulness of these tests in terms of sensitivity and specificity.

Methods and material: In March to May 2014, ten experienced shoulder surgeons were asked to name the SPE tests they would use for 12 pre-selected shoulder pathologies. A literature search on Pubmed.com and on the Cochrane library was conducted to assess the sensitivity and specificity of the most reported SPE tests for each pathology to investigate specificity and sensitivity of the test.

Results: In total 49 SPE tests were named. Nine surgeons reported at least one common SPE test for eight of 12 pathologies. For the following four pathologies; eight surgeons agreed upon one test for one pathology and for the remaining three pathologies six surgeons recommended one common test. The sensitivity and specificities of the 12 chosen tests were allowable.

Conclusion: There was a high degree of agreement on which SPE to use for which shoulder pathology and the specificity and sensitivity of the most reported SPE tests for each pathology were both acceptable. However, there is no clear evidence to support one SPE test over another in the literature.
\end{abstract}

*Corresponding author: Ann Ganestam, Department of Orthopaedic Surgery, Copenhagen University Hospital, Hvidovre, Kettegård Allé 30, DK-2650, Denmark, Tel: +46 708572222; E-mail: ganestam@hotmail.com

Citation: Ganestam A, Attrup ML, Hølmich P, Barfod KW (2015) Evaluation of the Clinical Practice of Shoulder Examination among Ten Experienced Shoulder Surgeons. J Orthop Res Physiother 1: 008.

Received: June 30, 2015; Accepted: July 16, 2015; Published: July 28, 2015
Keywords: Guideline; Physical examination test; Sensitivity; Shoulder; Specificity

\section{Key Messages}

Although a standardized guideline for a complete shoulder examination is not available, experienced shoulder surgeons showed a high degree of agreement about which SPE tests should be used for 12 most frequent shoulder pathologies. The recommendation of experienced shoulder surgeons could therefore be used as a foundation for standardized guidelines.

\section{Introduction}

Effective treatment of shoulder pathologies depends on a correct diagnosis. To aid diagnosis at least 184 tests of Shoulder Physical Examination (SPE) have been described [1]. The large number of different tests and gap of knowledge may cause a communication problem between clinicians [2-4]. Large meta-analyses have not been able to suggest a standardized guideline shoulder examination $[5,6]$.

In the search of a standardized guideline for shoulder examination, we evaluated the clinical practice of shoulder examination among ten experienced shoulder surgeons. The aim was to identify the most used SPE tests and evaluate the usefulness of these tests in terms of sensitivity and specificity and present the preferred SPE used by experienced shoulder surgeons.

\section{Subjects and Methods}

The study was conducted as a questionnaire based survey. The questionnaire was sent to all shoulder surgeons at a specialized arthroscopic centre, Arthroscopic Centre Amager (ACA). ACA is a highly specialized centre for arthroscopic surgery. Ten surgeons are specialized in shoulder arthroscopy and are considered as experts in examination and treatment of shoulder diseases. The period of investigation was March to May 2014. Each of the ten participants received a questionnaire listing the following 12 pathologies and they were asked to name the SPE tests they usually apply to for each of the 12 pathologies. They were able to choose any SPE test they were familiar with and write in the questionnaire, no description to the tests were given to the surgeons. Subsequently, we performed a thorough literature search on Pubmed.com and the Cochrane library to investigate the sensitivity and specificity of the most reported test for each of the following 12 pathologies.

The 12 pathologies were: 1) Acromioclavicular joint arthrosis, 2) Subacromial impingement syndrome, 3) Tear/lesion of the supraspinatus tendon, 4) Tear/lesion of the infraspinatus/teres minor tendon, 5) Tear/lesion of the subscapularis tendon, 6) Caput longum biceps pathology, 7) Superior Labrum Anterior-Posterior (SLAP) lesion, 8) Anterior instability, 9) Posterior instability, 10) Multi Directional Instability (MDI), 11) Adhesive capsulitis, and 12) Scapula alatae.

\section{Results}

Ten out of 10 (100\%) shoulder surgeons completed the questionnaire. The mean experience as shoulder surgeon was 10 years (range, 4-22 years). A total of 49 different SPE tests were reported. For 
Citation: Ganestam A, Attrup ML, Hølmich P, Barfod KW (2015) Evaluation of the Clinical Practice of Shoulder Examination among Ten Experienced Shoulder Surgeons. J Orthop Res Physiother 1: 008.

- Page 2 of 5 •

\begin{tabular}{|c|c|c|}
\hline Pathology & ShPE & $\mathbf{N}$ \\
\hline \multirow{4}{*}{ Acromiocavicular joint arthrosis } & Examination with direct palpitation & 10 \\
\hline & Crossover test & 9 \\
\hline & O'Brien's test & 3 \\
\hline & Springing test & 1 \\
\hline \multirow{3}{*}{ Subacromial impingement syndrome } & Hawkins Kennedy test & 10 \\
\hline & De Neers test without local anaesthesia & 7 \\
\hline & Painful arc test & 6 \\
\hline \multirow{10}{*}{ Tears/lesion of the supraspinatus tendon } & Jobe test & 9 \\
\hline & Drop arm & 5 \\
\hline & Ultra sound & 3 \\
\hline & External Rotation Lag Sign (ERLS) & 2 \\
\hline & Reduced active abduction & 2 \\
\hline & Inspection/atrophy & 1 \\
\hline & Beer drinker & 1 \\
\hline & Lag sign & 1 \\
\hline & Hawkin-Kennedy test & 1 \\
\hline & Impingement test & 1 \\
\hline \multirow{5}{*}{ Tears/lesion of the infraspinatus/teres major tendon } & Resisted external rotation & 9 \\
\hline & Lag sign & 3 \\
\hline & Ultra sound & 3 \\
\hline & Infraspinatus drop arm test & 2 \\
\hline & Inspection/atrophy & 1 \\
\hline \multirow{7}{*}{ Tears/lesion of the subscapularis tendon } & Belly press test & 9 \\
\hline & Lift-off & 8 \\
\hline & Lag sign & 4 \\
\hline & Push-off & 1 \\
\hline & Increased passive external rotation & 1 \\
\hline & Bear hug & 1 \\
\hline & Internal rotation of 0 degrees & 1 \\
\hline \multirow{7}{*}{ Caput longum biceps pathology } & Speeds' test & 8 \\
\hline & O'Brien's test & 5 \\
\hline & Examination with direct palpation & 4 \\
\hline & Yergason's test & 3 \\
\hline & Biceps Load II test & 1 \\
\hline & Pain when using the biceps muscle & 1 \\
\hline & Elbow flexion with resistance & 1 \\
\hline \multirow{7}{*}{ Superior Labrum Anterior and Posterior (SLAP) lesion } & O'Brien's test & 9 \\
\hline & Speeds' test & 3 \\
\hline & Apprehension test & 1 \\
\hline & Upper cut test & 1 \\
\hline & Proximalisering of caput & 1 \\
\hline & Yergason test & 1 \\
\hline & Biceps load test & 1 \\
\hline \multirow{4}{*}{ Anterior Instability } & Apprehension test & 10 \\
\hline & Relocation test & 9 \\
\hline & Load'n'shift test & 3 \\
\hline & Hyper abduction test & 1 \\
\hline \multirow{2}{*}{ Posterior Instability } & Posterior apprehension test & 6 \\
\hline & Load'n'shift test & 3 \\
\hline
\end{tabular}


Citation: Ganestam A, Attrup ML, Hølmich P, Barfod KW (2015) Evaluation of the Clinical Practice of Shoulder Examination among Ten Experienced Shoulder Surgeons. J Orthop Res Physiother 1: 008.

- Page 3 of 5 •

\begin{tabular}{|c|c|c|}
\hline & Jerks test & 2 \\
\hline & Pull up test & 1 \\
\hline & Jahnke test & 1 \\
\hline & AP-translocation test & 1 \\
\hline & Kim's test & 1 \\
\hline \multirow{5}{*}{ Multi Direction Instability (MDI) } & Anterior posterior-translocation & 7 \\
\hline & Sulcus Sign & 6 \\
\hline & Passive glenohumeral abduction & 1 \\
\hline & Drawers test & 1 \\
\hline & Downward pull & 1 \\
\hline \multirow{2}{*}{ Adhesive capsulitis } & Reduced Passive Range of Motion (PROM) & 10 \\
\hline & Anterior posterior-translocation & 1 \\
\hline \multirow{4}{*}{ Scapula alatae } & Wall push up & 6 \\
\hline & Inspection of shoulder rotation & 4 \\
\hline & Repeated flexion & 3 \\
\hline & Kiblers manoeuvre & 2 \\
\hline
\end{tabular}

Table 1: Shows the distribution of Shoulder Physical Examination (SPE) tests that were reported by the ten shoulder surgeons to diagnose shoulder pathologies. $\mathrm{N}=$ number of shoulder surgeons who used the SPE test

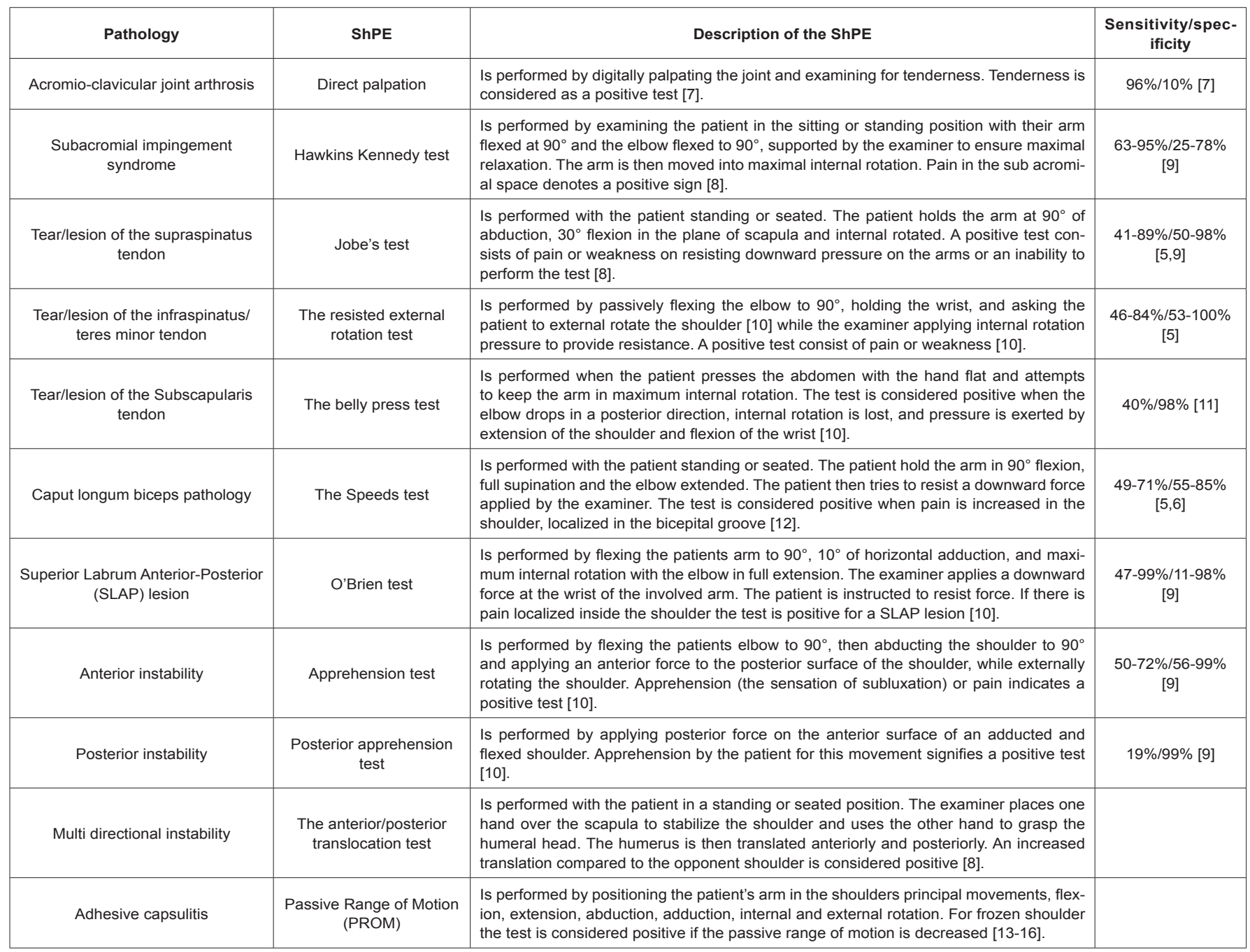




\begin{tabular}{|c|c|c|}
\hline Scapula alatae & Wall push up test & $\begin{array}{l}\text { Is performed with the patient leaning up against a wall (or down on the floor) with the } \\
\text { hands outstretched, facing the wall. The patient is then asked to lean closer towards } \\
\text { the wall flexing the elbows and then push the body back, until once again in a standing } \\
\text { position. While performing this test the patient's scapula is observed for movement. If } \\
\text { there is rotation or winging of scapula, the test is considered positive [10]. }\end{array}$ \\
\hline
\end{tabular}

Table 2: The 12 chosen pathologies and the Shoulder Physical Examination (SPE) tests used by most shoulder surgeons are described. For each test the sensitivity and specificity reported in the literature is shown.

eight out of 12 pathologies at least one SPE test that was used by at least nine out of ten surgeons (Table 1). Description of and sensitivity and specificity for the most reported SPE test for each pathologies is presented in table 2.

\section{Discussion}

The large number of different Shoulder Physical Examination (SPE) tests could contribute to a problem in communication, as clinicians tend to mix up the tests [2]. The purpose of this study was to identify the most used SPE tests, among 10 experienced shoulder specialists, for 12 of the commonest shoulder pathologies and to evaluate the usefulness of these tests in terms of sensitivity and specificity. Perhaps, this may be the foundation for a future standardized guideline for shoulder examinations.

Of the more than 184 SPE tests described, 49 were reported by our study population. For eight of 12 pathologies, there was a high degree of accordance as it was possible to identify an SPE test preferred by 9 to 10 of the experts [1]. For the remaining four pathologies, there was a moderate degree of accordance as it was possible to identify an SPE test preferred by 6 to 8 of the experts.

In the examination for acromioclavicular joint arthrosis, there was consensus on the usage of direct palpation as ten out of ten surgeons used this test. It has shown a high sensitivity (96\%) but a low specificity (10\%) [7]. In other words, a positive test is a poor indicator of presence of the pathology whereas the negative test is a strong indicator of absence of the pathology.

In the examination for subacromial impingement syndrome, there was consensus on the usage of Hawkins-Kennedy test as ten out of ten surgeons used this test. In the literature, the pooled sensitivity was $79 \%$ and the specificity $59 \%$ [9]. However, in the meta-analysis by Hegedus et al., a Diagnostic Odds Ratio (DOR) around one was reported suggesting a limited usefulness of the test [9]. It has been suggested that the Hawkins-Kennedy test can be used as a screening test of impingement symptoms rather than identifying the pathologic mechanism [8].

In the examination for tears of supraspinatus tendon, there was consensus on the usage of Jobe's test as nine out of ten surgeons used it. The sensitivity ranged from $41 \%$ to $81 \%$ and the specificity ranged from $50 \%$ to $98 \%$ [5]. A positive test is a rather strong indicator of presence of the pathology and a negative test a rather strong indicator of absence of the pathology [9].

In the examination for tears of infraspinatus/teres major tendon, consensus was on the resisted external rotation test, which was used by nine out of ten surgeons. The sensitivity ranged from $46 \%$ to $84 \%$ and the specificity from $53 \%$ to $100 \%$ [9]. Again, a positive test is a rather strong indicator of presence of the pathology and a negative test a rather strong indicator of absence of the pathology.

In the examination for tears of subscapularis tendon, there was consensus on the Belly press test as nine out of ten surgeons used it. The sensitivity was found to be $40 \%$ and the specificity $98 \%$ [11]. This means that a positive test is a very strong indicator of presence of the pathology whereas a negative test is a modest indicator of absence of the pathology.

In the examination for pathology of the long head of biceps usage of speeds test was recommended by eight out of ten surgeons. The test has shown a sensitivity ranging from $49-71 \%$ and a specificity ranging from 55 to $87 \%[5,6]$. As such, a positive test is a moderate indicator of presence of the pathology and a negative test is a moderate indicator of absence of the pathology. Hegedus et al., recommended the test for examining SLAP lesions rather than pathology of the long head of biceps, whereas Calis et al., recommended the use of Speeds test before use of other SPE tests $[6,9]$.

In the examination for Superior Labrum Anterior and Posterior (SLAP) lesions, there was consensus on the usage of O'Brien test, which was used by nine out of ten surgeons. Sensitivity ranging from $47 \%$ to $99 \%$ and specificity ranging from $11 \%$ to $98 \%$ has been found [9]. If ignoring the only study with both high sensitivity and high specificity [9] a positive test seems to be a moderate indicator of presence of the pathology and a negative test a strong indicator of absence of the pathology.

In the examination for anterior instability ten out of ten surgeons reported the apprehension test. Sensitivity ranging from $50 \%$ to $72 \%$ and specificity ranging from $56 \%$ to $99 \%$ has been found [9]. Hegedus et al., found the studies of great quality, but without sufficient power to be incorporated in a meta-analysis [9].

In the examination for posterior instability, six out of ten surgeons used the posterior apprehension test. A pooled sensitivity of 19\% and specificity of $99 \%$ have been found [5]. As such, a positive test is a very strong indicator of presence of the pathology whereas the negative test is a poor indicator of absence of the pathology.

In the examination for Multi Direction Instability (MDI), the most frequently used test was anterior posterior-translocation test as seven out of ten surgeons used it. Extra notice should be taken to discrepancy between instability and laxity. Laxity of the shoulder varies between patients and laxity found by an SPE does not automatically imply that the patient has instability [17].

In the examination for adhesive capsulitis there was consensus on the usage of reduced Passive Range of Motion (PROM) in all directions as ten out of ten surgeons used it. Full passive range of motion in any plane suggests another diagnosis than adhesive capsulitis [13-16]. In other words, this test can be used to exclude the diagnosis rather than confirm it.

In the examination for scapula alatae, six out of ten surgeons reported the wall push up test. For MDI, scapula alatae and adhesive capsulitis we were not able to find any data examining the sensitivity or specificity.

The sensitivity and specificity varied a lot between the 12 chosen SPE tests with sensitivity ranging from $19 \%$ to $99 \%$ and the specificity from $10 \%$ to $100 \%$. Considering this, one could ask if it is reasonable to recommend a standardized guideline based on the chosen tests. A standardized guideline has its clear advantages as it allows for better 
communication between healthcare professionals and it is a potential aid for inexperienced investigators. On the downside it might limit investigators in pursuing the test that feels best in their hands and it might reduce specificity if people use one instead of numerous tests to investigate for a given pathology [6]. Taken into account that several SPE tests had a high specificity; it might be advantageous to perform a set of two or three SPE tests for each pathology. It does however not seem realistic to expect general practitioners and general physiotherapist to remember between 24 and 36 different tests to do a shoulder examination.

On this basis we find it reasonable to recommend the 12 chosen SPE tests as a minimum repertoire for a good basic shoulder examination or as a general screening for shoulder pathology. Shoulder surgeons, sports physicians, sports physiotherapist and others with a special interest in physical shoulder examination will increase the specificity of their examinations by using additional tests.

\section{Limitations}

The study is vulnerable to selection-bias as all surgeons were from the same department and as such inevitably influenced by a local culture. Eight of ten surgeons have had prior experience and training from other hospitals before joining this department and the department does not have a general recommended guideline for shoulder examination. The questionnaire also constitute a limitation since the surgeons where asked to describe which exams they were using for a give disease entity, not for a complete shoulder examination. We are aware that the experts usually use more than one test for a given disease entity for a complete examination.

\section{Conclusion}

There was a high degree of agreement on which SPE to use for which shoulder pathologies and the specificity and sensitivity of the most reported SPE tests for each pathologies is acceptable.

However, there is no clear evidence to support one SPE test over another. In order to enhance communication between health care professionals we have highlighted one SPE test for each of the 12 shoulder pathologies. We recommend the use of these 12 chosen SPE tests (Table 2) as a minimum repertoire for a shoulder examination. There is need for better studies examining validity and accuracy for SPE tests and their correlation to treatment outcome.

\section{References}

1. http://www.larsblond.com/skulderkompendium.11.html\#Skulderkompendium
2. de Winter AF, Jans MP, Scholten RJ, Devillé W, van Schaardenburg D, et al. (1999) Diagnostic classification of shoulder disorders: interobserver agreement and determinants of disagreement. Ann Rheum Dis 58: 272-277.

3. Liesdek C, van der Windt DA, Koes BW, Bouter LM (1997) Soft-tissue disorders of the shoulder. Physiotherapy 83: 12-17.

4. Nomden JG, Slagers AJ, Bergman GJ, Winters JC, Kropmans TJ, et al. (2009) Interobserver reliability of physical examination of shoulder girdle. Man Ther 14: 152-159.

5. Hegedus EJ, Goode AP, Cook CE, Michener L, Myer CA, et al. (2012) Which physical examination tests provide clinicians with the most value when examining the shoulder? Update of a systematic review with meta-analysis of individual tests. Br J Sports Med 46: 964-978.

6. Caliș M, Akgün K, Birtane M, Karacan I, Caliș H, et al. (2000) Diagnostic values of clinical diagnostic tests in subacromial impingement syndrome. Ann Rheum Dis 59: 44-47.

7. Walton J, Mahajan S, Paxinos A, Marshall J, Bryant C, et al. (2004) Diagnostic values of tests for acromioclavicular joint pain. J Bone Joint Surg Am 86: 807-812.

8. Cools AM, Cambier D, Witvrouw EE (2008) Screening the athlete's shoulder for impingement symptoms: a clinical reasoning algorithm for early detection of shoulder pathology. Br J Sports Med 42: 628-635.

9. Hegedus EJ, Goode A, Campbell S, Morin A, Tamaddoni M, et al. (2008) Physical examination tests of the shoulder: a systematic review with meta-analysis of individual tests. Br J Sports Med 42: 80-92.

10. Donnelly TD, Ashwin S, Mac Farlane RJ, Waseem M (2013) Clinical assessment of the shoulder. Open Orthop J 7: 310-315.

11. Barth JR, Burkhart SS, De Beer JF (2006) The bear-hug test: a new and sensitive test for diagnosing a subscapularis tear. Arthroscopy 22: 1076-1084.

12. Guanche CA, Jones DC (2003) Clinical testing for tears of the glenoid labrum. Arthroscopy 19: 517-523.

13. Ewald A (2011) Adhesive capsulitis: A review. Am Fam Physician 83: 417422.

14. Robinson CM, Seah KT, Chee YH, Hindle P, Murray IR (2012) Frozen shoulder. J Bone Joint Surg Br 94: 1-9.

15. Kelley MJ, McClure PW, Leggin BG (2009) Frozen shoulder: evidence and a proposed model guiding rehabilitation. J Orthop Sports Phys Ther 39: 135148.

16. Neviaser AS, Hannafin JA (2010) Adhesive capsulitis: a review of current treatment. Am J Sports Med 38: 2346-2356.

17. Mallon WJ, Speer KP (1995) Multidirectional instability: current concepts. J Shoulder Elbow Surg 4: 54-64. 